\title{
Edge minimization in de Bruijn graphs
}

\author{
Uwe Baier*, Thomas Büchler*, Enno Ohlebusch*, Pascal Weber* \\ *Institute of Theoretical Computer Science \\ Ulm University, D-89069 Ulm, Germany \\ \{uwe.baier, thomas . buechler, enno.ohlebusch, pascal-1.weber\}@uni-ulm.de
}

\begin{abstract}
This paper introduces the de Bruijn graph edge minimization problem, which is related to the compression of de Bruijn graphs: find the order- $k$ de Bruijn graph with minimum edge count among all orders. We describe an efficient algorithm that solves this problem. Since the edge minimization problem is connected to the BWT compression technique called "tunneling", the paper also describes a way to minimize the length of a tunneled BWT in such a way that useful properties for sequence analysis are preserved. Although being a restriction, this is significant progress towards a solution to the open problem of finding optimal disjoint blocks that minimize space, as stated in Alanko et al. (DCC 2019).
\end{abstract}

\section{Introduction}

De Bruijn graphs play an important role in string processing. Originally invented for solving combinatorial problems [1, de Bruijn graphs have also been used in bioinformatics, e.g. in genome assembly [2] or to describe variations between different strings [3]. As de Bruijn graphs that show variations between genomes can get very large, there have been attempts to compress such graphs by merging nodes [4].

In this paper, we will present a similar compression scheme for such graphs with the difference that we view the de Bruijn graph as a multigraph in which every overlapping of two consecutive $k$-mers in the underlying cyclic string induces an edge in the graph. Then, instead of merging nodes, we fuse the edges between two nodes $x$ and $y$ if $y$ is the only successor of $x$ and $x$ is the only predecessor of $y$. We also introduce the de Bruijn graph edge minimization problem, asking for the order $k$ such that an edge-reduced de Bruijn graph of an underlying string has the minimum amount of edges under all possible orders of the graph.

The main contribution of our paper is the presentation of an algorithm which, given a special trie, is capable of solving the de Bruijn graph edge minimization problem with a worst-case time that is linear on the minimum amount of edges. The algorithm is designed in such a way that it can be implemented with an FM-index [5, 6] in an overall run-time of $O(n \log \sigma)$, where $\sigma$ is the alphabet size.

We also show a direct connection between edge reduction in de Bruijn graphs and a recent BWT compression technique called tunneling [7]. It will be shown that each edge-reduced de Bruijn graph corresponds to a tunneled BWT of the underlying string, where the number of edges in the graph is identical to the length of the tunneled BWT. Therefore, we show that solving the edge minimization problem provides significant progress towards a solution to the open problem of finding the optimal disjoint blocks that minimize space, as stated in [8]. 
Finally, the paper describes how the outputs of the edge minimization algorithm can be used to produce the associated tunneled BWT. We provide a publicly available implementation of our algorithms [9] and present results for the edge reduction ratio and tunneled BWT size ratio for real world data coming from the Pizza \& Chili text corpus [10] and the Repetitive corpus [11].

\section{Preliminaries}

We start with some standard string notation. Throughout this paper, any interval $[i, j]$ is meant to be an interval over the natural numbers and indices start with 1 , except when stated differently.

Let $\Sigma$ be an ordered alphabet of size $\sigma=|\Sigma|$. A string $S$ of length $n=|S|$ over $\Sigma$ is a finite sequence of $n$ characters from $\Sigma$. Throughout this paper, we assume that $S$ is null-terminated, i.e. that it ends with the EOF-symbol $\$$ (which is the smallest symbol in $\Sigma)$. For $i, j \in[1, n], S[i]$ denotes the $i$-th character of $S$ and $S[i . . j]$ denotes the substring of $S$ starting at the $i$-th and ending at the $j$-th position. A length $k$ substring of $S$ is called a $k$-mer. The empty string with length 0 is denoted by $\varepsilon$. Let $S$ and $T$ be two strings of length $n$ and $m$ over a common alphabet $\Sigma$. We write $S<_{\text {lex }} T$ if $S$ is lexicographically smaller than $T$, i.e. $S$ is a proper prefix of $T$ or there exists a $k \in[1, \min \{n, m\}]$ with $S[1 . . k-1]=T[1 . . k-1]$ and $S[k]<T[k]$.

Next, the de Bruijn graph (DBG) shall be defined. Note that our definition differs from common definitions as [12] or [13] because we consider de Bruijn graphs originating from cyclic strings.

Definition 1. Let $S$ be a string of length $n$ and $k \in[1, n]$. If we concatenate the $k$-mer prefix of $S$ to $S$ itself, then we obtain the string $Z_{k}(S):=S[1 . . n] S[1 . . k]$. Furthermore, the set of all $k$-mers of $Z_{k}(S)$ is

$$
\mathcal{K}:=\left\{Z_{k}(S)[i . . i+k-1] \mid i \in[1, n]\right\} .
$$

The de Bruijn graph $G_{k}(S)=(\mathcal{K}, E)$ of order $k$ is a directed multigraph, where the multiset of edges is defined as (the superscript $m$ denotes the multiplicity of an edge):

$$
E:=\left\{(x[1 . . k], x[2 . . k+1])^{m} \mid x \in \Sigma^{k+1} \text { occurs exactly } m \text { times in } Z_{k}(S)\right\} .
$$

An example of a DBG can be found in Figure 1, It should be noted that, independent of the order $k$, each DBG $G_{k}(S)$ contains exactly $|S|$ edges due to the definition of the string $Z_{k}(S)$. The next definition introduces the rotation-trie $R T(S)$ built from all rotations (cyclic shifts) of $S$.

Definition 2. Let $S$ be a string of length $n$. The rotation-trie $R T(S)$ is a trie [14] built from the rotations $S[1 . . n], S[2 . . n] S[1], \ldots, S[n] S[1 . . n-1]$ of $S$ such that the following holds for any two nodes $\phi$ and $\varphi$ of the trie: If $\phi$ is the left sibling of $\varphi$, then its labe $]^{1} l(\phi)$ is lexicographically smaller than the label of $\varphi$, i.e. $l(\phi)<_{\text {lex }} l(\varphi)$.

\footnotetext{
${ }^{1}$ The label of a node $\phi$ in a trie emerges by concatenating all edge labels in the path from the root node to node $\phi$.
} 


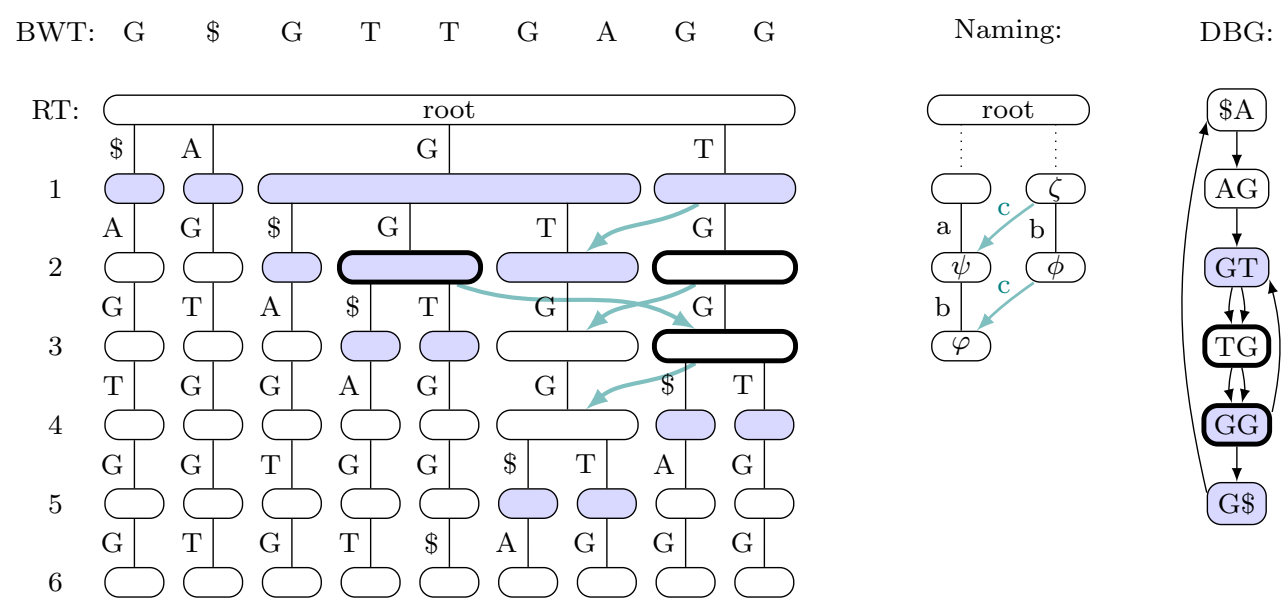

Figure 1: Left: First 6 levels of the rotation-trie $R T(S)$ of the string $S=$ AGTGGTGG\$. Nodes with siblings are filled. Teal-colored arrows represent unique Weiner links (with the connected nodes having size $>1$ ). The nodes of which incoming edges can be fused in the DBG are drawn bold-framed. Middle: Greek letters for nodes are used as depicted here. Right: Order-2 de Bruijn graph $G_{2}(S)$.

We furthermore denote by $l b(\phi)$ resp. $r b(\phi)$ the lexicographic rank of the leftmost resp. rightmost leaf of $\phi$ among all leaves of $R T(S)$, and by $|\phi|$ the number of leaves of the subtree rooted at $\phi$, i.e. $|\phi|=r b(\phi)-l b(\phi)+1$. With $R T_{k}(S)$ we denote the set of nodes at level $k$ in $R T(S)$. $R T_{\leq k}(S)$ denotes the nodes in the levels 1 to $k$.

Figure 1 shows an example of a rotation-trie. Note that all the rotations of a null-terminated string are distinct, which implies that $R T(S)$ has $n=|S|$ leaves. To see the connection between RTs and DBGs we need the definition of so-called Weiner links [15].

Definition 3. Let $R T(S)$ be a rotation-trie and let $\phi$ be a node of $R T(S)$. We call the set

$W L:=\{(\phi, \varphi, c) \mid \varphi$ is a node of $R T(S)$ such that for some $c \in \Sigma$

$$
l(\varphi)=c l(\phi) \text { or }|l(\phi)|=|S| \text { and } l(\varphi)=c l(\phi)[1 . .|S|-1]\}
$$

the set of Weiner links of node $\phi$. A Weiner link $(\phi, \varphi, c) \in W L$ is called unique if there exists no other Weiner link $(\phi, \psi, d) \in W L$ with $\varphi \neq \psi$.

We state the following connections between de Bruijn graphs and rotation-tries: let $G_{k}(S)=(\mathcal{K}, E)$ be a DBG of order $k$ for a string $S$, and let $R T(S)$ be the rotationtrie of the same string $S$. It can be seen that the $k$-mers in $\mathcal{K}$ correspond to the nodes in $R T_{k}(S)$, i.e. for every node $y \in \mathcal{K}$ there is exactly one node $\phi$ in $R T_{k}(S)$ with $l(\phi)=y$.

Lemma 1. Let $k<n$. There is an edge $(x, y)^{m} \in E$ in $G_{k}(S)=(\mathcal{K}, E)$ with $c=x[1]$ if and only if there is a Weiner link $(\phi, \varphi, c)$ in $R T(S)$ such that (a) $l(\phi)=y,(b)$ $l(\varphi)=c y$, and (c) $m$ is the number of leaves $\vartheta$ in the subtree rooted at $\phi$ with $l(\vartheta)[n]=c$ (i.e. the last character in the label of the leaf is $c)$. 
Proof. ' $\Rightarrow$ ' If $(x, y)^{m} \in E$ and $c=x[1]$, then by definition the string $z=c y$ (of length $k+1$ ) occurs exactly $m$ times in $Z_{k}(S)$. Obviously, there are nodes $\phi$ and $\varphi$ in $R T(S)$ such that $l(\phi)=y$ and $l(\varphi)=c y$. Hence there is Weiner link $(\phi, \varphi, c)$. Consider a leaf $\vartheta$ in the subtree rooted at $\phi$ with $l(\vartheta)[n]=c$. The string $l(\vartheta)$ is a rotation of $S$. If we cyclically shift the last character $c$ of $l(\vartheta)$ to the front, then we obtain another rotation of $S$ that has $c y$ as a prefix. It is not difficult to see that there are $m$ occurrences of $c y$ in $Z_{k}(S)$ if and only if there a $m$ rotations of $S$ that have $c y$ as a prefix. This proves the claim.

' $\Leftarrow$ ' Suppose there is a Weiner link $(\phi, \varphi, c)$ in $R T(S)$ such that (a) $l(\phi)=y$, (b) $l(\varphi)=c y$, and (c) $m$ is the number of leaves in the subtree rooted at $\phi$ with $l(\vartheta)[n]=$ c. This implies that the string $z=c y$ occurs exactly $m$ times in $Z_{k}(S)$. Thus, $(x, y)^{m} \in E$.

\section{Edge minimization in de Bruijn graphs}

In this section, we will introduce the DBG edge minimization problem. Edge reduction between two nodes $x, y \in \mathcal{K}$ in a DBG $G_{k}(S)$ is defined as follows: if $y$ is the only successor of $x$ and $x$ is the only predecessor of $y$, then the number of edges between $x$ and $y$ can be reduced to one.

Definition 4. Let $G_{k}(S)=(\mathcal{K}, E)$ be a DBG for string $S$ of order $k$. Denote by

$F:=\left\{(x, y)^{m} \in E \mid y\right.$ is the only successor of $x$ and $x$ is the only predecessor of $\left.y\right\}$

the set of fusible edges. The edge-reduced de Bruijn graph $\tilde{G}_{k}(S)=(\mathcal{K}, \tilde{E})$ of $G_{k}(S)$ is defined by decreasing the multiplicity of all fusible edges to one, that is,

$$
\tilde{E}:=(E \backslash F) \uplus\left\{(x, y)^{1} \mid(x, y)^{m} \in F\right\}
$$

An example of edge-reduced DBGs can be found in Figure 2, Definition 4 is closely related to so-called compressed de Bruijn graphs [4], with the difference that in Definition 4 edges are fused while in compressed DBGs nodes are merged. We are now able to state the edge minimization problem.

Problem 5. Given a string $S$ of length $n$, the de Bruijn graph edge minimization problem asks for finding an order $k \in[1, n]$ such that the amount of edges in the edge-reduced DBG $\tilde{G}_{k}(S)=(\mathcal{K}, \tilde{E})$ is minimal, that is, find the order $k$ such that

$$
k=\underset{\tilde{k} \in[1, n]}{\operatorname{argmin}}\left\{\sum_{(x, y)^{m} \in \tilde{E}} m \mid \tilde{G}_{\tilde{k}}(S)=(\mathcal{K}, \tilde{E}) \text { is the order } \tilde{k} \text { edge-reduced DBG of } S\right\}
$$

Figure 2 illustrates the problem. At first sight, it seems that the problem is easily solvable: The higher the order gets, the more nodes exist, resulting in fewer branching edges and therefore in more edge fusions. Then, at a high enough order $k$, the amount of nodes prevents edge fusions because there exist not enough edges between nodes. Summing these observations up, one could think that the amount of edges in an edgereduced DBG follows a parabolic-shaped function depending on the order $k$, where 

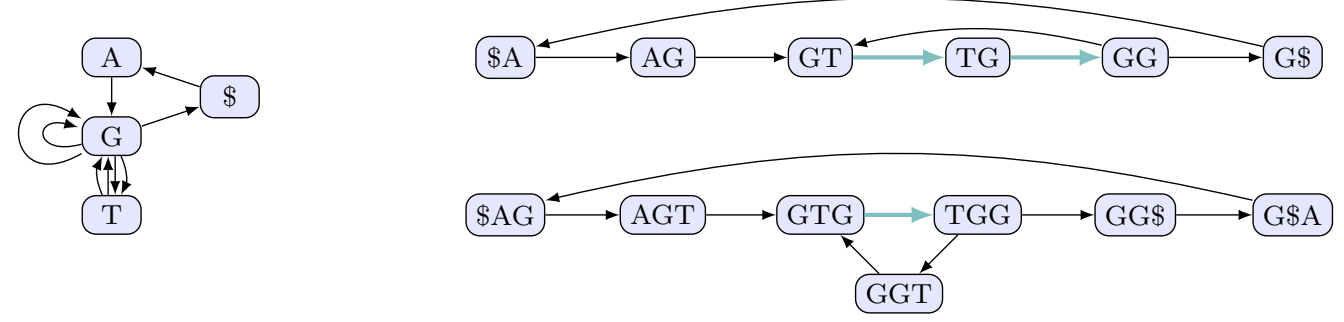

Figure 2: Edge-reduced de Bruijn graphs with order $k=1$ (left), $k=2$ (upper right) and $k=3$ (lower right) built from the string AGTGGTGG\$. Fused edges are indicated with teal arrows, the DBG with order $k=2$ contains the least edges, namely $9-2=7$ edges.

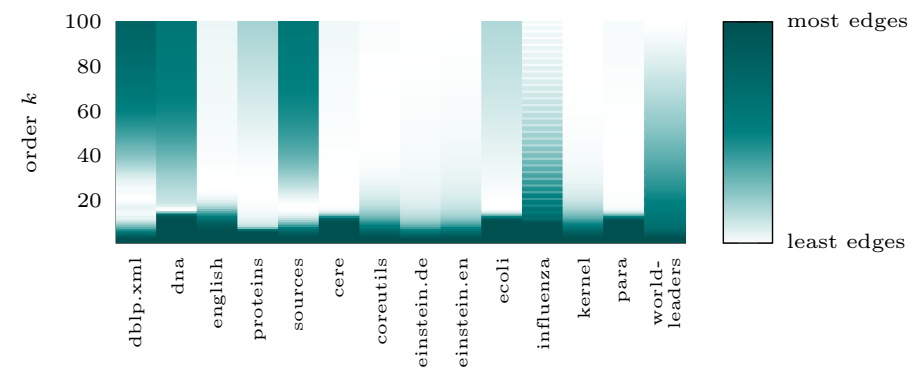

Figure 3: Matrix plot showing for each test file the relative amount of edges depending on the order $k$ of the edge-reduced DBG.

the only local minimum coincides with the global minimum. Unfortunately, as Figure 3 shows, this observation does not hold true for all strings: the file influenza clearly shows that the function can contain multiple local minima, so we need more advanced techniques to compute the order $k$ such that $\tilde{G}_{k}(S)$ has a minimum amount of edges.

\section{Naive computational approach}

It is a consequence of Lemma 1 that the set of predecessors of a node $y$ in the graph $G_{k}(S)$ (the set of nodes $x \in \mathcal{K}$ with $(x, y)^{m} \in E$ ) can be determined as follows: Find the node $\phi$ in $R T(S)$ that corresponds to $y$ (i.e. $l(\phi)=y$ ) and follow its Weiner links. For each Weiner link $(\phi, \varphi, c)$, the parent node $\psi$ of $\varphi$ is a predecessor of $x$ in $G_{k}(S)$ (more precisely, $x=l(\psi)$ is a predecessor). The next lemma is the basis of our algorithms.

Lemma 2. A node $\phi$ in $R T(S)$ has a unique Weiner link $(\phi, \varphi, c)$ and $\varphi$ has no siblings if and only if $x=l(\psi)$ is the only predecessor of $y=l(\phi)$, where $\psi$ is the parent node of $\varphi$ in $G_{k}(S)$, and $y$ is the only successor of $x$.

Proof. It follows directly from Lemma 1 that $\phi$ has a unique Weiner link $(\phi, \varphi, c)$ if and only if $x=l(\psi)$ is the only predecessor of $y=l(\phi)$ in $G_{k}(S)$, where $\psi$ is the parent node of $\varphi$ in $R T(S)$. Suppose that $\phi$ has a unique Weiner link $(\phi, \varphi, c)$. We must show that $\varphi$ has no siblings (or equivalently, its parent $\psi$ has only one child) if and only if $y=l(\phi)$ is the only successor of $x=l(\psi)$. Let $b=y[k]$ be the last character of $y$.

' $\Rightarrow$ ' If $\varphi$ has no siblings, then every occurrence of $x$ in $Z_{k}(S)$ is followed by the 
character $b$. Therefore, $y$ is the only successor of $x$.

' $\Leftarrow$ ' If $\varphi$ has a sibling $\vartheta$, then there is an occurrence of $x$ in $Z_{k}(S)$ that is followed by the character $l(\vartheta)[k]=d \neq b$. Therefore, $x$ has a least two successors.

Definition 6. Let $\phi$ be a node at level $k$ in $R T(S)\left(\phi \in R T_{k}(S)\right)$. Define

$$
w_{k}(\phi)= \begin{cases}1 & \text { if } \phi \text { has a unique Weiner link }(\phi, \varphi, c) \text { and } \varphi \text { has no siblings } \\ |\phi| & \text { otherwise }\end{cases}
$$

Lemma 3. Let $m_{k}$ be the number of edges in the edge-reduced de Bruijn graph $\tilde{G}_{k}(S)$. Then $m_{k}=\sum_{\phi \in R T_{k}(S)} w_{k}(\phi)$.

Proof. Let $\phi$ be a node in $R T_{k}(S)$. The weight $w_{k}(\phi)$ equals the number of incoming edges to node $y=l(\phi)$ in $\tilde{G}_{k}(S)$ : If $\phi$ has a unique Weiner link $(\phi, \varphi, c)$ and $\varphi$ has no siblings, then the corresponding edge $(x, y)^{m}$ in $G_{k}(S)$ is fusible, and its multiplicity is reduced to 1 in $\tilde{G}_{k}(S)$. Otherwise, all the $|\phi|$ incoming edges to $y$ are not fusible.

Using Lemma 3, we are able to describe a naive solution to the edge minimization problem. We traverse $R T(S)$ level-wise. At level $k$, we compute the weight $w_{k}(\phi)$ of each node $\phi$ at this level by checking whether or not $\phi$ has a unique Weiner link $(\phi, \varphi, c)$ and $\varphi$ has no siblings. The sum of these weights yields $m_{k}$, the number of edges in $\tilde{G}_{k}(S)$ and $\tilde{k}=\operatorname{argmin}_{k \in[1, n]}\left\{m_{k}\right\}$ is the solution we are looking for. The drawback of this method is that all nodes of $R T(S)$ must be visited. Since there are $O\left(n^{2}\right)$ many nodes in $R T(S)$, the resulting worst-case time complexity of this method is $O\left(n^{2}\right)$, which is not acceptable for big data.

\section{Edge minimization algorithm}

To improve the run-time of the naive computational approach, we will update the amount of edges level by level, where levels are visited from top to bottom.

Lemma 4. For every path $p$ from the root to a leaf in $R T(S)$, there is exactly one node $\phi$ in $p$ so that (1) $\phi$ has a unique Weiner link, (2) the ancestors of $\phi$ in $p$ have multiple Weiner links, and (3) every node in the subtree rooted at $\phi$ has a unique Weiner link.

Proof. Let $\phi$ be the first node on a path from the root to a leaf $\vartheta$ that has a unique Weiner link ( $\vartheta$ has a unique Weiner link, so $\phi$ must exist). Let $c$ be the last character in the label of $\vartheta$, i.e. $l(\vartheta)[n]=c$. Since $\phi$ has a unique Weiner link, the last character in the label of each leaf in the subtree rooted at $\phi$ must be $c$. This implies (3) and proves the lemma.

Lemma 5. Let $\zeta$ be a node in $R T(S)$ at level $k<n$ that has only one child $\phi$.

1. If $\zeta$ has a unique Weiner link, then $w_{k+1}(\phi)=1$.

2. If $\zeta$ has multiple Weiner links, then $w_{k+1}(\phi)=w_{k}(\zeta)$. 


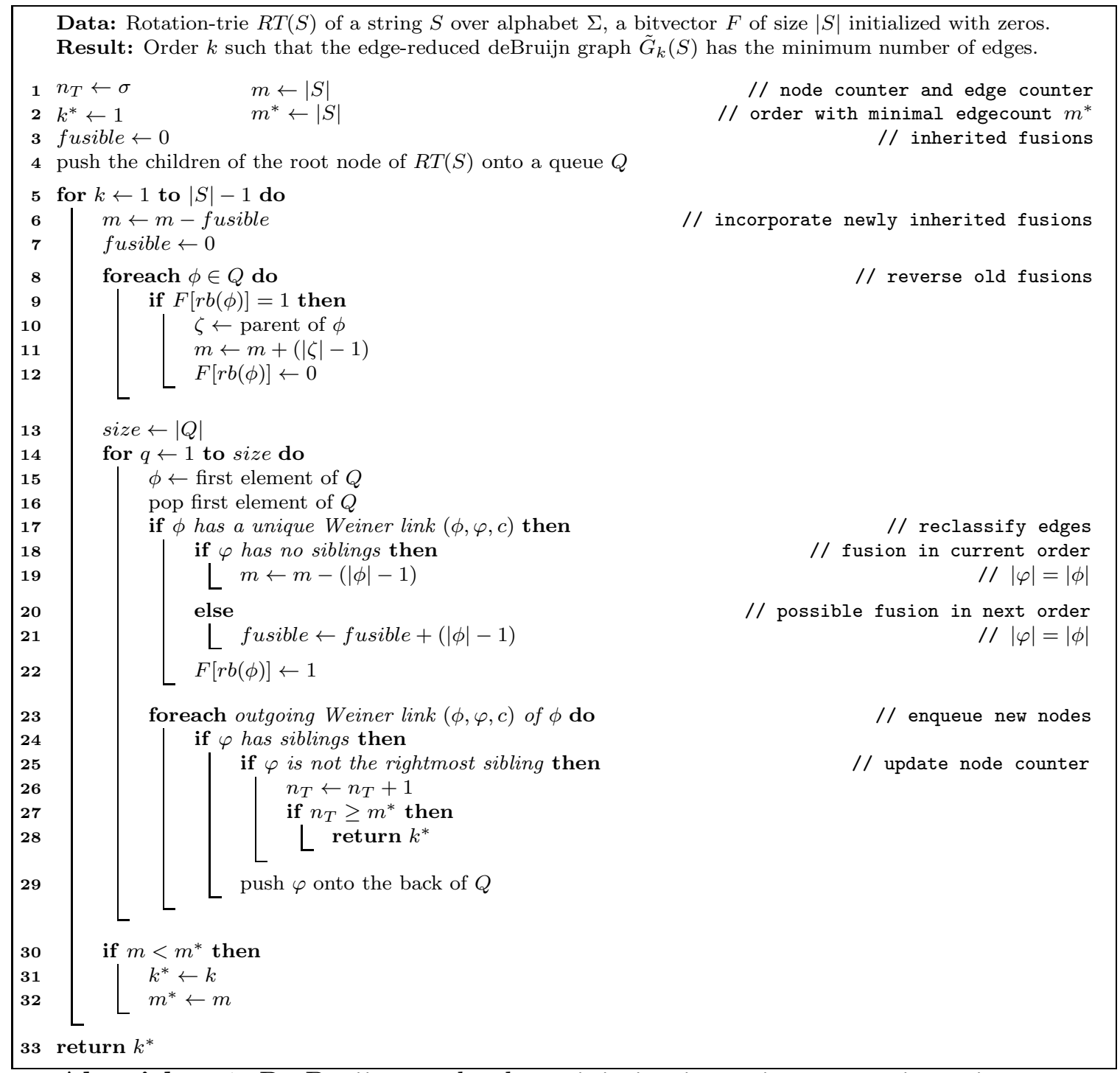

Algorithm 1: De Bruijn graph edge minimization using a rotation-trie.

Proof. (1) If $\zeta$ has a unique Weiner link, then $\phi$ has a unique Weiner link $(\phi, \varphi, c)$. It is easy to see that in this case $\phi$ is the only child of its parent if and only if $\varphi$ is the only child of its parent. Thus, $\varphi$ has no siblings and $w_{k+1}(\phi)=1$ by Definition 6 . (2) If $\zeta$ has multiple Weiner links, then so does its only child $\phi$. By Definition 6 , $w_{k}(\zeta)=|\zeta|$ and $w_{k+1}(\phi)=|\phi|$. Now the claim follows from $|\zeta|=|\phi|$.

According to the preceding lemma, for every node $\phi$ in $R T(S)$ at level $k+1$ that has no siblings, we can infer $w_{k+1}(\phi)$ if we know the number of Weiner links of its parent $\zeta$ as well as $w_{k}(\zeta)$. Moreover, a node $\phi$ that has no siblings inherits the weight from its parent $\zeta$ except for the special case $*$ in which $\zeta$ has a unique outgoing Weiner link $(\zeta, \psi, c)$ and $\psi$ has multiple siblings (in this case $w_{k}(\zeta)=|\zeta|$ and $w_{k+1}(\phi)=1$ ).

These observations lead to an efficient incremental algorithm, which calculates $m_{k}$ from $m_{k-1}$ by a case distinction on the differences between the edge-reduced de Bruijn graphs $\tilde{G}_{k-1}(S)$ and $\tilde{G}_{k}(S)$. The final implementation (Algorithm 2) does not 
efficiently support the operation 'How many children does a node have?' That is why Algorithm 1 does not use this operation.

At the moment, we will ignore lines 25-28 of the algorithm. Before we will prove the correctness of Algorithm 1, we exemplify the different cases in the algorithm by considering the nodes T, TG, TGG, and TGG\$ in the example of Figure 1, In our considerations, we will make use of the following fact (see Theorem 7): Before the body of the outer for-loop is executed for a value $k$, the queue $Q$ contains exactly those nodes of $R T(S)$ at level $k$ that have siblings (nodes that have no sibling are treated implicitly by the observations above). In the execution of the for-loop for $k=1$, it is detected that node $\mathrm{T}$ has a unique Weiner link to GT, but GT has siblings. Thus, the multiple edges from $\mathrm{G}$ to $\mathrm{T}$ in the DBG of order 1 cannot be fused. In line 21, the variable fusible is incremented by $|T|-1(|T|$ denotes the number of leaves in the subtree rooted at $\mathrm{T}$ ) and the rightmost leaf in the subtree rooted at $\mathrm{T}$ is marked (i.e. $F[r b(T)]=1$ ). The variable fusible will be used to take care of the special case *. From that point in time, it is clear that TG, TGG, and TGG\$ have unique Weiner links.

Before the for-loop is executed for $k=2$, the queue $Q$ contains the nodes AG, G\$, $\mathrm{GG}$, and GT. In line $6, m=m_{1}$ is decremented by the value of fusible. Recall that the contribution of node $\mathrm{T}$ to fusible was $|T|-1$. Now, what is the contribution of node TG to $m_{2}$ ? Obviously, TG has a unique Weiner link pointing to GTG because $\mathrm{T}$ has a unique Weiner link pointing to GT. Furthermore, TG has siblings if and only if GTG has siblings. We infer from TG $\notin Q$ that TG has no siblings, hence neither GTG does. Consequently, $w_{2}(T G)=1$ because the multiple edges from GT to TG in the DBG of order 2 can be fused. The difference between the weights of $\mathrm{T}$ and TG is $w_{1}(T)-w_{2}(T G)=|T|-1$. This value was (correctly) subtracted from $m=m_{1}$. What would happen if (fictitiously) the node TG were in $Q$ ? In this case, GTG would have siblings (since TG has siblings) and the subtraction of $|T|-1$ from $m$ must be compensated by adding $|T|-1$ to $m$. This would be done in the loop in lines 8 to 12 . If the node TG were in $Q$, then its parent $\mathrm{T}$ would have multiple children, and all of its children would be in $Q$ because they have siblings. For the rightmost child $\vartheta$ of $\mathrm{T}$, it would follow $r b(\vartheta)=r b(T)$. Since $F[r b(T)]=1$, the value $w_{2}(T G)-1$ would be added to $m$ in line 11 (and $F[r b(T)]$ would be set to 0 ).

Before the for-loop is executed for $k=3$, the queue $Q$ contains the nodes GG\$ and GGT. Node TGG has a unique Weiner link pointing to node GTGG because TG has a unique Weiner link pointing to GTG. The fact that TGG is not in $Q$ means that GTGG has no siblings. Therefore, the multiple edges from GTG to TGG in the DBG of order 3 can be fused and $w_{3}(T G G)=1=w_{2}(T G)$. Since $w_{2}(T G)$ is already included in the value of $m$, there is no need to change $m$ w.r.t. node TGG (i.e. given $m_{2}$, the incremental computation of $m_{3}$ does not need to consider this case explicitly). We say that TGG (implicitly) inherits its weight from its parent TG.

Before the for-loop is executed for $k=4$, the queue $Q$ contains the nodes TGG\$ and TGGT. Node TGG\$ has a unique Weiner link pointing to node GTGG $\$$ because TGG has a unique Weiner link pointing to GTGG. However, TGG\$ has siblings because it is contained in $Q$. In this case, $w_{3}(T G G)$ cannot be inherited and $m$ must be updated. The change from $m_{3}$ to $m_{4}$ w.r.t. node TGG and its children $\vartheta_{1}, \ldots, \vartheta_{q}$ 
can be computed by adding (a) $|T G G|-1$ to $m$ (this corresponds to the situation in which none of the nodes $\vartheta_{1}, \ldots, \vartheta_{q}$ admits a fusion) and (b) subtracting $\left|\vartheta_{i}\right|-1$ from $m$ for each child $\vartheta_{i}$ of node TGG that admits a fusion. (a) will be done in lines 8 to 12 because $\vartheta_{q} \in Q$ and (b) will be done in the for-loop starting at line 14. Let us prove the correctness of Algorithm 1 .

Theorem 7. Algorithm 1 maintains the following invariants:

1. Before the body of the for-loop starting at line 5 is executed for a value $k$, the queue $Q$ contains exactly those nodes of $R T_{k}(S)$ that have siblings.

2. After the body of the for-loop starting at line 5 was executed for a value $k$, the variable $m$ stores the value $m_{k}$.

3. Before the body of the for-loop starting at line 5 is executed for a value $k$, the variable $n_{T}$ stores the value $\left|R T_{k}(S)\right|$.

Proof. For $k=1$ property 1 holds true because initially all nodes of $R T_{1}(S)$ (the children of the root node) are pushed onto $Q$ (and there are at least two because of the EOF-symbol). So let $k \geq 2$. Lines 23-29 of Algorithm 1 push a node $\varphi$ onto $Q$ only if $\varphi$ has siblings. So we must prove that all nodes with siblings are pushed onto $Q$. For an indirect proof, suppose that there is a node $\varphi \in R T_{k}(S)$ that has siblings but is not pushed onto $Q$. Let $(\phi, \varphi, c)$ be its incoming Weiner link. Clearly, if $\varphi$ has siblings, so does $\phi$. By induction, we may assume that before the for-loop is executed for the value $k-1$, the queue $Q$ contains exactly those nodes of $R T_{k-1}(S)$ that have siblings. Consequently, at that point in time, $\phi$ is in $Q$. Hence $\varphi$ will be pushed onto $Q$ in lines 23-29. This contradiction proves that property 1 is an invariant.

In order to show that property 2 is also invariant, we may assume by induction that $m=m_{k-1}$ before the for-loop is executed for $k$ (initially $m=m_{0}=|S|$, i.e. there are no fusions). We will show by a case distinction that after the execution of the body of the for-loop for $k$, the value of $m$ equals $m_{k}$. Let $\phi$ be a node in $R T(S)$ at level $k$ and let $\zeta$ be its parent node.

1. $\phi$ has no siblings (or equivalently, $\phi \notin Q$ ). In this case, $\phi$ has a unique Weiner link if and only if $\zeta$ has a unique Weiner link.

(a) $\phi$ has a unique Weiner link $(\phi, \varphi, c)$.

In this case, $w_{k}(\phi)=1$ because $\varphi$ has no siblings. Furthermore, $\zeta$ has a unique Weiner link to the parent $\psi$ of $\varphi$. If $\psi$ has no siblings, then $w_{k-1}(\zeta)=1$ and $\phi$ inherits its weight from its parent. Otherwise, the parent $\psi$ of $\varphi$ has siblings. In this special case $*$, the variable fusible was incremented by $|\zeta|-1$ in the previous iteration of the for-loop, and this value was subtracted from $m$ in the current iteration.

(b) $\phi$ has multiple Weiner links.

In this case, $\zeta$ has multiple Weiner links. Since $|\zeta|=|\phi|$, it follows that $w_{k-1}(\zeta)=|\zeta|=|\phi|=w_{k}(\phi)$. That is, $\phi$ inherits its weight from its parent.

2. $\phi$ has siblings (or equivalently, $\phi \in Q$ ). 
(a) If $F[r b(\zeta)]=1$, then there is an ancestor $\alpha$ of $\zeta$ such that (i) $r b(\alpha)=r b(\zeta)$, (ii) the variable fusible was incremented by $|\alpha|-1$ in a previous iteration $i$ of the for-loop, (iii) $F[r b(\alpha)]=F[r b(\zeta)]$ was set to 1 in iteration $i$, (iv) the value $|\alpha|-1$ was subtracted from $m$ in iteration $i+1$, and $(\mathrm{v}) F[r b(\zeta)]=1$ was not reset to 0 from iteration $i+1$ to the current iteration. It follows as a consequence that node $\zeta$ inherited its weight from its ancestors in those iterations and therefore $|\zeta|=|\alpha|$. Note that $\phi$ and all its siblings are in $Q$. Since only its rightmost sibling $\vartheta$ is marked (i.e. $F[r b(\vartheta)]=1),|\zeta|-1=|\alpha|-1$ is added once to $m$ in line 11. In summary, the subtree rooted at $\zeta$ contributes the value $|\zeta|$ to $m$. This corresponds to the situation in which no child of $\zeta$ admits a fusion. Since $\phi$ and all its siblings are in $Q$, possible fusions of the children of $\zeta$ are taken care of by lines 17-19.

(b) If $F[r b(\zeta)]=0$, then the subtree rooted at $\zeta$ contributes the value $|\zeta|$ to $m=$ $m_{k-1}$ and this value is not changed in the current iteration. The remaining arguments are the same as in the preceding case.

We finally show property 3 holds. For $k=1$ it is true, because $R T_{1}(S)$ contains a node for each letter of $\Sigma$ and $n_{T}$ is initialized with the value $\sigma$. We further show that $n_{T}$ will be correctly updated in a loop iteration. Therefore we have a look on the changes between $\left|R T_{k}(S)\right|$ and $\left|R T_{k+1}(S)\right|$. The number of nodes in $R T_{k+1}(S)$ equals the number of children of the nodes in $R T_{k}(S)$. Each node in $R T_{k}(S)$ has exactly one rightmost child (a single child is rightmost). So the amount of nodes changes by the number of children that are not rightmost. If a node is a not rightmost child, it has siblings and therefore it will be added to the queue in line 29 due to property 1. Right before that $n_{T}$ will be incremented by one (line 25-26). Hence exactly the children that are not rightmost cause an incrementation of $n_{T}$ during the for-loop, the variable stores the correct value at the begin of the next iteration.

Next, we will explain lines 25-28 of Algorithm 1. As the node of each edge-reduced DBG must have at least 1 outgoing edge, the number of edges in such a graph must be greater than or equal to its number of nodes. This means that there are at least $\left|R T_{k}(S)\right|$ edges in the edge-reduced DBG of order $k$. Furthermore, the number of nodes per level is increasing from top to bottom $\left(\left|R T_{k}(S)\right| \leq\left|R T_{k+1}(S)\right|\right.$ for all $\left.k\right)$. When it is clear that the next level will contain $n_{T} \geq m^{*}$ nodes, the algorithm terminates and returns $k^{*}$. This is correct because the current level then must have at least $n_{T} \geq m^{*}$ edges (each node in the next level has at least one incoming Weiner link) and each further level must have at least $n_{T}$ nodes and thus at least $m^{*}$ edges.

Theorem 8. Given the rotation-trie $R T(S)$ and a zero-initialized bitvector F, Algorithm 1 has a worst-case time complexity of $O\left(m^{*}\right)$.

Proof. We will use an amortized analysis to show the theorem. It is assumed that the rotation-trie $R T(S)$ is given and that the following operations are supported in constant time:

- determine the (size of the) parent of a node (lines 10-11) 


\begin{tabular}{|c|c|c|c|}
\hline$\varphi \in M$ & $\varphi$ has a right sibling & $\varphi$ is rightmost sibling & $\varphi$ has no sibling \\
\hline$\varphi \in M \cap R T_{\leq k}(S)$ & \multirow{2}{*}{$M_{1}$} & $M_{2}$ & $M_{4}$ \\
\cline { 1 - 3 } & & \multicolumn{2}{|c|}{$M_{3}$} \\
\hline
\end{tabular}

Table 1: partition of $M$

- generate Weiner links (lines 17 and 23)

- check whether a node $\varphi$ has siblings (lines 18 and 24):

- if a node $\varphi$ has siblings, check whether $\varphi$ is the rightmost sibling (line 25)

Let $M$ be the set of all nodes $\varphi \in R T(S)$ such that there is a Weiner link $(\phi, \varphi, c)$ generated by Algorithm 1. It is clear that the run-time of the algorithm is proportional to $|M|$. Let $m^{*}$ and $k$ be the values of the variables upon termination of Algorithm 1. We will show that $|M| \leq 4 m^{*}$. It is clear that $M \subseteq R T_{\leq k+1}(S)$, because the algorithm terminates in the $k$-th iteration (in which nodes of the $k+1$ level of $R T(S)$ are generated). We partition $M$ into four disjoint subsets $M_{1}, \ldots, M_{4}$ and show that each subset is of size $\leq m^{*}$. The definition of the subsets is depicted in Table 1. The size of $M_{1}$ is exactly $m^{*}$ because of lines 25-28 of Algorithm 1. Each node in $M_{2}$ has at least one left sibling and this sibling is in $M_{1}$, hence $\left|M_{2}\right| \leq\left|M_{1}\right|=m^{*}$. Next, we show that $\left|M_{3}\right| \leq m^{*}$. Since $n_{T}=\left|R T_{k}(S)\right|$ at the end of the $(k-1)$ th iteration and the algorithm did not terminate in this iteration (i.e. $n_{T}<m^{*}$ ), it follows that $\left|R T_{k}(S)\right|<m^{*}$. Each node in $R T_{k}(S)$ has exactly one child $\varphi$ that has no right sibling (either $\varphi$ has no siblings or $\varphi$ is the rightmost sibling). So the set $\left\{\varphi \mid \varphi \in R T_{k+1}(S)\right.$ and $\varphi$ has no right sibling $\}$ has a size $<m^{*}$. Since this is a superset of $M_{3}$, it follows that $\left|M_{3}\right|<m^{*}$.

Finally, we show that $\left|M_{4}\right| \leq\left|R T_{k}(S)\right|$ (the reader should be aware of the fact that this is by no means obvious). The theorem then follows from $\left|R T_{k}(S)\right|<m^{*}$, which was shown above. With each node $\varphi \in M_{4}$, we associate the lexicographically smallest node $\theta \in R T_{k}(S)$ such that $l(\varphi)$ is a suffix of $l(\theta)$. We claim that if $\varphi \in M_{4}$ is associated with $\theta \in R T_{k}(S)$, then there is no other node $\varphi^{\prime} \in M_{4}$ that is associated with $\theta$. To prove the claim, suppose to the contrary that there are two distinct nodes $\varphi, \varphi^{\prime} \in M_{4}$ which are associated with $\theta$. So both $l(\varphi)$ and $l\left(\varphi^{\prime}\right)$ are suffixes of $l(\theta)$. W.l.o.g. we may assume that $|l(\varphi)|<\left|l\left(\varphi^{\prime}\right)\right|$ because $\varphi \neq \varphi^{\prime}$. It follows as a consequence that $l(\varphi)$ is a proper suffix of $l\left(\varphi^{\prime}\right)$, hence $l\left(\varphi^{\prime}\right)=c^{q} \ldots c^{1} l(\varphi)$ for a $q \geq 1$ and $c^{1}, \ldots, c^{q} \in \Sigma$. Let $\varphi_{i}$ be the node with label $c^{i} \ldots c^{1} l(\varphi)$ for $1 \leq i \leq q$. Note that $\varphi^{\prime}=\varphi_{q}$. Since $\varphi$ has no sibling, it will not be pushed onto the queue $Q$. Therefore, the Weiner link $\left(\varphi, \varphi_{1}, c_{1}\right)$ will not be generated and thus $\varphi_{1}$ will not be pushed onto the queue $Q$ either. By iterating this argument, we conclude that the Weiner link $\left(\varphi_{q-1}, \varphi_{q}, c_{q}\right)$ will not be generated. So $\varphi^{\prime} \notin M$ and this implies $\varphi^{\prime} \notin M_{4}$. This contradiction shows that distinct nodes $\varphi, \varphi^{\prime} \in M_{4}$ are associated with distinct nodes $\theta^{\prime}, \theta \in R T_{k}(S)$. Since each node $\varphi \in M_{4}$ is associated with a node $\theta \in R T_{k}(S)$, it follows $\left|M_{4}\right| \leq\left|R T_{k}(S)\right|$. 


\section{Implementation with an FM-index}

To implement Algorithm 1, we need a framework that (1) allows us to traverse a rotation-trie level by level in a top-down fashion and (2) supports the operations listed in the preceding section (in the run-time analysis of Algorithm 1). The work of Beller et al. [16] provides such a framework (although this is non-obvious at first sight). To be precise, they developed an algorithm that enumerates all LCP-intervals in a top-down manner; see also [17, Algorithm 7.19]. Their algorithm uses the wavelet tree of the Burrows-Wheeler transform of string $S$ [5].

Recall that the Burrows-Wheeler transform computes the string $L$ for a nullterminated string $S$ of length $n$ as follows:

1. Form a conceptual matrix $M^{\prime}$ whose rows are the rotations (cyclic shifts) of $S$.

2. Compute the matrix $M$ by sorting the rows of $M^{\prime}$ lexicographically.

3. Output the last column $\mathrm{L}$ of $M$.

The Burrows-Wheeler transform can be generalized to multiple strings.

If $\omega$ is a substring of $S$, then the $\omega$-interval is the largest interval $[i, j]$ of $M$ such that $\omega$ is a prefix of the rotations of $S$ in the interval $[i, j]$. In what follows, for each character $c, C[c]$ is the overall number of occurrences of characters in $\mathrm{L}[1, n]$ that are strictly smaller than $c$. Given the $\omega$-interval $[l b, r b]$ and a character $c$, the $c \omega$-interval $[i, j]$ can be computed by $i=C[c]+\operatorname{rank}_{c}(\mathrm{~L}, l b-1)+1$ and $j=C[c]+\operatorname{rank}_{c}(\mathrm{~L}, r b)$, where $\operatorname{rank}_{c}(\mathrm{~L}, l b-1)$ returns the number of occurrences of character $c$ in the prefix $\mathrm{L}[1, l b-1]$ (we have $i \leq j$ if $c w$ is a substring of $S$; otherwise $i>j$ ); see [18] for details. The (balanced) wavelet tree [19] of $\mathrm{L}$ supports such a backward search step in $O(\log \sigma)$ time, where $\sigma$ is the size of the alphabet. Backward search can be generalized on the wavelet tree as follows: Given an $\omega$-interval $[l b, r b]$, a slight modification of the procedure getIntervals $(l b, r b)$ described in [20] returns the list $[(c,[i, j]) \mid c \omega$ is a substring of $S$ and $[i, j]$ is the $c \omega$-interval], where the first component of an element $(c,[i, j])$ is a character. The worst-case time complexity of the procedure getIntervals is $O(o c c+o c c \cdot \log (\sigma / o c c))$, where occ is the number of elements in the output list; see [21, Lemma 3]. Thus, the time per generated interval is $O(\log \sigma)$.

In Algorithm 2, a node $\phi$ is represented by the interval $[l b(\phi), r b(\phi)]$. Since for each $k$ the intervals of all nodes in $R T_{k}(S)$ (the nodes of level $k$ ) are a partition of the interval $[1, n]$, we can represent $R T_{k}(S)$ by marking all the right boundaries of the node intervals in a bitvector of size $n$. For technical reasons, we use a bitvector $B$ of size $n+1$ in Algorithm 2, in which $B[1]$ and $B[n+1]$ are always set to 1 . Given a level $k, B[r b(\phi)+1]$ is set to 1 for all nodes $\phi \in R T_{k}(S)$. Fix some node $\zeta \in R T_{k-1}(S)$ and let $\phi \in R T_{k}(S)$ be its rightmost child. Since $r b(\zeta)=r b(\phi), B[r b(\zeta)+1]=1$ must hold at both levels $k-1$ and $k$. In other words, the representation of the next higher level can be obtained by just adding ones for the boundaries of new nodes (line 14). Algorithm 2 maintains the following invariant: when the body of the for-loop starting at line 8 is executed for a value $k$ and line 23 is reached, then the boundaries of all nodes in level $k$ are marked in $B$. 
Given a node $\phi$ in level $k$, all its Weiner links $(\phi, \varphi, c)$ can be generated by the procedure call getIntervals $(l b(\phi), r b(\phi))$ because $[l b(\phi), r b(\phi)]$ is the $l(\phi)$-interval and the call returns the set $M=\{(c,[i, j]) \mid c \in \Sigma$ and $[i, j]$ is the $c l(\phi)$-interval $\}$. The set $M$ contains just one element if and only if there is a unique Weiner link $(\phi, \varphi, c)$. The following statements hold true:

1. Node $\varphi$, represented by the interval $[l b(\varphi), r b(\varphi)]$, has no siblings if and only if $B[l b(\varphi)]=1$ and $B[r b(\varphi)+1]=1$.

2. If $\varphi$ has a sibling, then $\varphi$ is the rightmost sibling if and only if $B[r b(\varphi)+1]=1$.

To prove (1), consider the parent node $\psi$ of $\varphi$. Since $\psi$ is a node at level $k$, we have $B[l b(\psi)]=1, B[r b(\psi)+1]=1$, and $B[p]=0$ for all $p$ with $l b(\psi)<p<r b(\psi)+1$. Furthermore, $l b(\psi) \leq l b(\varphi)<r b(\varphi)+1 \leq r b(\psi)+1$ because $\varphi$ is a child of $\psi$. If $\varphi$ has no siblings, then $l b(\varphi)=l b(\psi)$ and $r b(\varphi)=r b(\psi)$, which implies $B[l b(\varphi)]=1$ and $B[r b(\varphi)+1]=1$. If $\varphi$ has siblings, then $l b(\varphi) \neq l b(\psi)$ or $r b(\varphi) \neq r b(\psi)$. Hence $B[l b(\varphi)]=0$ or $B[r b(\varphi)+1]=0$. The simple proof of $(2)$ is left to the reader.

Algorithm 2 must also be able to determine the parent of a node. That is why it traverses the nodes of a level in lexicographical order (i.e. from left to right). To this end, it employs $\sigma$ queues (a queue $Q_{c}$ for each $c \in \Sigma$ ). Algorithm 2 maintains the following invariant: before the body of the for-loop starting at line 8 is executed for a value $k$, the queue $Q_{c}$ stores the intervals $[l b(\phi), r b(\phi)]$ of all nodes $\phi \in R T_{k}(S)$, that have siblings and whose labels starts with $c$, in lexicographical order. This is certainly true for $k=1$ because of the initialization of the queues in lines 6-7: the procedure call getIntervals $(1, n)$ returns the set $\{(c,[i, j]) \mid c \in \Sigma$ and $[i, j]$ is the $c$-interval $\}$ and each queue contains just one element. Moreover, if it is for $k$, it remains true for $k+1$ because the foreach-loop starting at line 25 processes the characters of $\Sigma$ in alphabetical order. Since a queue $Q_{c}$ contains only nodes with siblings and these are in lexicographical order, the leftmost child of a node is encountered first in the foreach-loop starting at line 11, followed by some or no 'middle children', followed by the rightmost child. When the first child is encountered, its left boundary, which is also the left boundary of its parent, is stored in the variable last (line 17). The right boundary of the parent is identical to right boundary of the rightmost child. Therefore, the rightmost child $\phi$ is reached when $B[r b(\phi)+1]=1$ (line 18). At this moment, we know the left and right boundary of the parent, which gives us its size (line 20). In line 22, the variable last is reset (to ensure that it will be correctly set to a new left boundary, when the next leftmost child is encountered).

It follows as in the run-time analysis of Algorithm 1 that, given an FM-Index and two zero-initialized bitvectors, Algorithm 2 has a worst-case run-time of $\mathcal{O}\left(m^{*} \log \sigma\right)$. This improves the complete run-time from $O\left(n^{2}\right)$ (construction of the rotations-trie and Algorithm 1) to $O(n \log \sigma)$ (construction of the FM-index and Algorithm 2). 


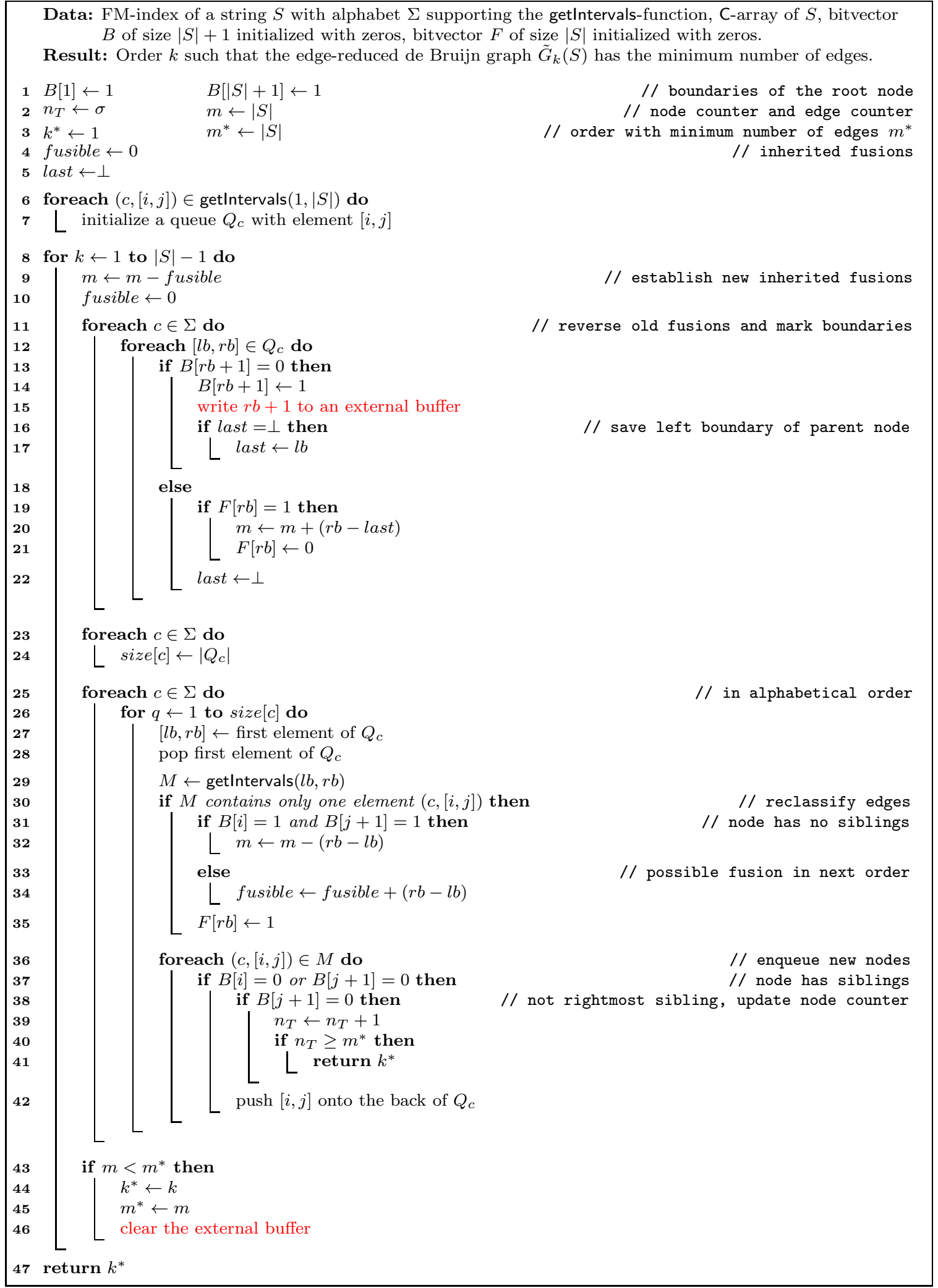

Algorithm 2: De Bruijn graph edge minimization using a FM index. 
So far we are able to compute the order $k$ such that the edge-reduced DBG $\tilde{G}_{k}(S)$ contains the minimum amount of edges. For typical applications, like e.g. compressed DBGs, we would like to have some information about the nodes themselves. This information typically consists of a bitvector $B$ where the boundaries of each node in the corresponding level in the BWT are marked. We could of course compute these boundaries by another breadth-first traversal of the trie until level $k$, but this seems unnecessary as Algorithm 2 already performs kind of a breadth-first traversal.

Indeed, note that Algorithm 2 computes the extra information during its execution by marking the left boundaries of each node. Unfortunately, once the best level of the trie has been processed, Algorithm 2 writes additional markings in $B$ until the algorithm terminates. To retain the node boundaries of the solution, we somehow have to get rid of the additional markings. This is the point where the red marked commands of Algorithm 2 (lines 15 and 46) come into play: Once we have processed the best level, line 43 is no longer executed, so the external buffer contains positions of all "unwanted" markings in $B$. Thus, to obtain the node boundaries of the solution, we just have to clear all markings at positions in the external buffer after Algorithm 2 terminates. As Algorithm 1 has an output-sensitive run-time of $O\left(\mathrm{~m}^{*}\right)$, the external buffer has a size of at most $O\left(m^{*}\right)$, and therefore computing the node boundaries requires $O\left(m^{*}\right)$ time.

\section{Connections to tunneling}

To give an application of the edge minimization approach in de Bruijn graphs, we show connections to a new BWT compression technique called "tunneling". The idea of tunneling is that, given adjacent rotations in $M$ preceded by equal strings, those equal preceding strings can be "tunneled", i.e. fuse the equal strings to just one string and thereby reduce the size of the BWT. The technique was presented in [7] and extended to Wheeler graphs in [8]. The technique relies on equal preceding strings, which we will call prefix intervals. 2

Definition 9. Let $S$ be a string of length $n, \mathrm{~L}$ be its corresponding BWT. The LF-mapping is a permutation of integers in range $[1, n]$ defined as follows:

$$
\mathrm{LF}[i]:=\mathrm{C}[\mathrm{L}[i]]+\operatorname{rank}_{\mathrm{L}[i]}(\mathrm{L}, i)
$$

We write $\operatorname{LF}^{x}[i]$ for the $x$-fold application of $\operatorname{LF}$, i.e. $\operatorname{LF}^{x}[i]:=\underbrace{\operatorname{LF}[\operatorname{LF}[\cdots \operatorname{LF}[i] \cdots]] \text {, }}_{x \text { times }}$
define $\operatorname{LF}^{0}[i]:=i$ and the inverse of $\operatorname{LF}$ as $\operatorname{LF}^{-1}$.

Definition 10. Let $S$ be a string of length $n$ and let $\mathrm{L}$ be its BWT. A prefix interval is a pair of an integer $w \geq 0$ and an interval $[i, j] \subseteq[1, n](\mathrm{a}\langle w,[i, j]\rangle$ prefix interval) such that

$$
\mathrm{L}\left[\operatorname{LF}^{x}[i]\right]=\mathrm{L}\left[\operatorname{LF}^{x}[i+1]\right]=\cdots=\mathrm{L}\left[\operatorname{LF}^{x}[j]\right] \text { for all }-1 \leq x<w
$$

\footnotetext{
${ }^{2}$ Older publications use the term block, but the term prefix interval seems clearer to us because a prefix interval is an interval with equal preceding strings.
} 
Definition 10 is a combination of definitions from $[7$ and 8 , because both together unleash the full power of tunneling a BWT. Also note that varying $x$ from -1 to $w-1$ ensures that lexicographically adjacent rotations begin with the same character. Next, we will specify what the fusion of a prefix interval means.

Definition 11. Let $S$ be a string of length $n$ and let $\mathrm{L}$ be its BWT. Furthermore, let $\left\langle w_{1},\left[i_{1}, j_{1}\right]\right\rangle, \cdots,\left\langle w_{k},\left[i_{k}, j_{k}\right]\right\rangle$ be a set of disjoint prefix intervals in $\mathrm{L}$, that is, the sets $N_{l}:=\left\{\operatorname{LF}^{x}[y] \quad \mid i_{l} \leq y \leq j_{l}, 0 \leq x \leq w_{l}\right\}$ are pairwise disjoint.

Given two bitvectors $D_{\text {out }}$ and $D_{\text {in }}$ of size $n$ initialized with ones, tunneling terms the following procedure:

1. For all $1 \leq l \leq k$, set

$$
D_{\text {in }}\left[i_{l}+1\right]=\cdots=D_{\text {in }}\left[j_{l}\right]=0 \text { and } D_{\text {out }}\left[\operatorname{LF}^{w_{l}}\left[i_{l}+1\right]\right]=\cdots=D_{\text {out }}\left[\operatorname{LF}^{w_{l}}\left[j_{l}\right]\right]=0 \text {. }
$$

2. For all $1 \leq l \leq k$, mark

$\mathrm{L}\left[\operatorname{LF}^{x}[y]\right], D_{\text {out }}\left[\operatorname{LF}^{x}[y]\right]$ and $D_{\text {in }}\left[\operatorname{LF}^{x+1}[y]\right]$ for all $i_{l}<y \leq j_{l}$ and $0 \leq x<w_{l}$.

3. remove all marked entries from $\mathrm{L}, D_{\text {out }}$ and $D_{\text {in }}$.

Tunneling indeed "fuses" the strings of a prefix interval: only the uppermost string (uppermost row) of the prefix interval remains unchanged, all other strings are removed by item 3 of Definition 11, see Figure 4 for an example. Analogously to the LF-mapping in a normal BWT, the generalized LF-mapping in a tunneled BWT is given by the formula

$$
i \leftarrow \operatorname{select}_{1}\left(D_{\text {out }}, \operatorname{rank}_{1}\left(D_{\text {in }}, \mathrm{C}[\mathrm{L}[i]]+\operatorname{rank}_{\mathrm{L}[i]}(\mathrm{L}, i)\right)\right) .
$$

Navigating in a tunneled BWT has one more specialty: if the start of a tunnel is reached, a tunnel entry offset has to be saved, which is used at the end of the tunnel to jump back to the correct entry, see [7] and [8] for more details.

We next want to establish a connection between edge-reduced de Bruijn graphs and tunneling. It shows that the paths of nodes with fusible edges in a DBG coincide with prefix intervals in a BWT.

Theorem 12. Let $G_{k}(S)=(\mathcal{K}, E)$ be a $D B G$ of some string $S$, and let $\mathrm{L}$ be the $B W T$ of $S$. Let $p_{1}=\left(x_{1,1}, x_{1,2}, \ldots, x_{1, w_{1}}\right), \ldots, p_{m}=\left(x_{m, 1}, \ldots, x_{m, w_{x}}\right)$ be all longest paths in $G_{k}(S)$ such that $x_{i, j+1}$ is the only successor of $x_{i, j}$ and $x_{i, j}$ is the only predecessor of $x_{i, j+1}$ for all $1 \leq i \leq m$ and $1 \leq j<w_{i}$.

1. Each path $p=\left(x_{1}, \ldots, x_{w}\right)$ corresponds to a prefix interval $\langle w-1,[i, j]\rangle$ in $\mathbf{L}$ where the number of edges between nodes in p equals $(w-1) \cdot(j-i+1)$.

2. The corresponding prefix intervals are disjoint.

Proof.

1. Each node $x$ of a path spans a consecutive $x$-interval $[i, j]$ in the BWT matrix $M$, as rotations prefixed with $x$ are adjacent in the matrix $M$. Let $x$ and $y$ be two adjacent nodes of $p$ with intervals $\left[i_{x}, j_{x}\right]$ and $\left[i_{y}, j_{y}\right]$. Because $x$ is the only predecessor of $y$ 

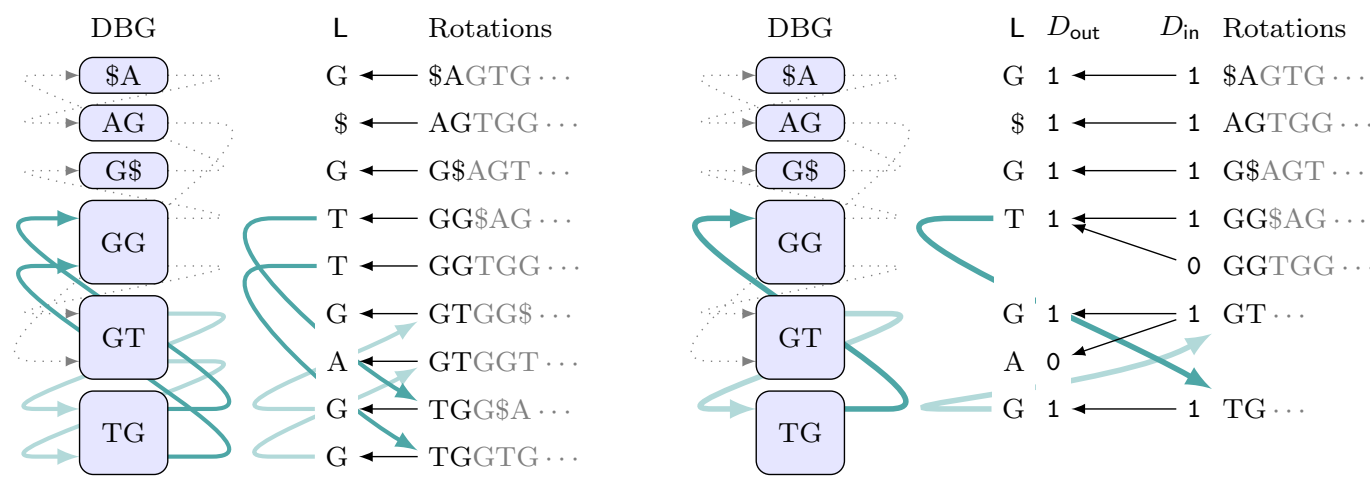

Figure 4: Correspondence between a de Bruijn graph with order $k=2$ and the BWT (left), and correspondence between the edge-reduced de Bruijn graph and the tunneled BWT (right) for $S=A G T G G T G G \$$. The left-hand side shows teal-colored fusible edges between nodes in the DBG and the corresponding LF-mappings in the BWT. The right-hand side shows the fused edges in the DBG, as well as the LF-mapping in the tunneled BWT by tunneling the $\langle 2,[4,5]\rangle$ prefix interval corresponding to the fusible path.

and $y$ is the only successor of $x$, the intervals have the same height $\left(j_{x}-i_{x}=j_{y}-i_{y}\right)$ and the entries in the BWT interval of the successor $y$ are equal $\left(\mathrm{L}\left[i_{y}\right]=\ldots=\mathrm{L}\left[j_{y}\right]\right)$, so $\left[\operatorname{LF}\left[i_{y}\right], \operatorname{LF}\left[j_{y}\right]\right]=\left[i_{x}, j_{x}\right]$ must hold. Because each edge from $x$ to $y$ corresponds to a text position where the $k$-mers overlap, there must be exactly $j_{y}-i_{y}$ edges between $x$ and $y$, so the path overall contains $(w-1) \cdot(j-i+1)$ edges.

2. As the paths are disjoint (otherwise one node would have multiple predecessors or successors), the intervals in $M$ and therefore the prefix intervals must be disjoint, too.

Theorem 12 states that edge-reducible paths in a DBG correspond to disjoint prefix intervals in the matching BWT, see also Figure 4. Moreover, the length of an edge-reduced DBG and the size of a tunneled BWT, obtained by tunneling the corresponding prefix intervals, is equal.

Corollary 13. Let $G_{k}(S)=(\mathcal{K}, E)$ be a $D B G$ of some string $S$, and let $\mathrm{L}$ be the $B W T$ of $S$. Let $p_{1}=\left(x_{1,1}, x_{1,2}, \ldots, x_{1, w_{1}}\right), \ldots, p_{m}=\left(x_{m, 1}, \ldots, x_{m, w_{x}}\right)$ be all longest paths in $G_{k}(S)$ such that $x_{i, j+1}$ is the only successor of $x_{i, j}$ and $x_{i, j}$ is the only predecessor of $x_{i, j+1}$ for all $1 \leq i \leq m$ and $1 \leq j<w_{i}$, and let $\left\langle w_{1}-1,\left[i_{1}, j_{1}\right]\right\rangle, \ldots,\left\langle w_{m}-1,\left[i_{m}, j_{m}\right]\right\rangle$ be the corresponding prefix intervals in $\mathrm{L}$.

Then, the size of the tunneled $B W T \mathrm{~L}, D_{\text {out }}, D_{\text {in }}$ obtained by tunneling $\left\langle w_{1}-1,\left[i_{1}, j_{1}\right]\right\rangle, \ldots,\left\langle w_{m}-1,\left[i_{m}, j_{m}\right]\right\rangle$ is equal to the number of edges in the edgereduced de Bruijn graph $\tilde{G}_{k}(S)=(\mathcal{K}, \tilde{E})$, that is, $|\mathrm{L}|=\sum_{(x, y)^{m} \in \tilde{E}} m$.

Proof. Initially, the number of edges as well as the size of the BWT is $|S|$. Edgereducing a path $p=\left(x_{1}, \ldots, x_{w}\right)$ with corresponding prefix interval $\langle w-1,[i, j]\rangle$ removes exactly $(w-1) \cdot(j-i+1)$ edges from $\tilde{G}_{k}(S)$. Analogously, tunneling the prefix interval $\langle w-1,[i, j]\rangle$ removes exactly $(w-1) \cdot(j-i+1)$ entries from $\mathrm{L}, D_{\text {out }}$ and $D_{\text {in }}$. 
Summing up, by combining the edge minimization problem with tunneling, we are able to produce a tunneled BWT which has minimum length under the restriction that only $k$-mer prefix intervals can be tunneled.

\section{Producing a tunneled BWT using the output of the edge minimization algorithm}

After establishing the connection between tunneling and edge minimization in de Bruijn graphs, we want to describe how Algorithm2 2 can be used to produce a tunneled $\mathrm{BWT}$. It shows that the node boundaries of the $k$-mers of the optimal order $k$ stored in the bitvector $B$ (Section "Retaining the node boundaries of solution") suffice to produce the corresponding tunneled BWT.

Looking at Definition [11 with the tunneling process, tunneling may be formulated alternatively as follows: Starting with two one-initialized bitvectors $D_{\text {in }}$ and $D_{\text {out }}$, set the start entries and all intermediate entries of each prefix interval in the bitvector $D_{\text {in }}$ to zero, except for the uppermost row of the prefix interval. Analogously, set the end entries and all intermediate entries of each prefix interval in $D_{\text {out }}$ to zero, except for the uppermost row of the prefix interval. Afterwards, remove all entries $\mathrm{L}[i]$ and $D_{\text {out }}[i]$ where $D_{\text {in }}[i]=0$ holds, as well as all $D_{\text {in }}[i]$-entries where $D_{\text {out }}[i]=0$ holds.

It is easy to show that the upper process produces the same tunneled BWT as the normal process from Definition [11. Also, the process does not regulate the order in which the columns $[i, j],[\operatorname{LF}[i], \operatorname{LF}[j]], \ldots,\left[\operatorname{LF}^{w}[i], \operatorname{LF}^{w}[j]\right]$ of a $\langle w,[i, j]\rangle$ prefix interval have to be marked.

Our approach thus is as follows: Given an interval $[i, j]$ of a $k$-mer $y$ in the BWT (those intervals can be obtained by the node boundary bitvector), we check if

1. $\mathrm{L}[i]=\cdots=\mathrm{L}[j]$. In this case, the $k$-mer $y$ has only one predecessor.

2. $[\operatorname{LF}[i], \operatorname{LF}[j]]$ are the boundaries of another $k$-mer $x$ (this can be checked using the node boundaries). In this case, $y$ is the only successor of $x$.

If both conditions are true, we know that edges between $x$ and $y$ can be fused. Combined with the tunneling process described above, we set $D_{\text {in }}[i+1]=\cdots=$ $D_{\text {in }}[j]=0$ and $D_{\text {out }}[\operatorname{LF}[i+1]]=\cdots=D_{\text {out }}[\operatorname{LF}[j]]=0$. Then, after processing each $k$-mer in this way, in a front-to-back scan, we remove all entries $\mathrm{L}[i]$ and $D_{\text {out }}[i]$ where $D_{\text {in }}[i]=0$ holds, as well as all $D_{\text {in }}[i]$-entries where $D_{\text {out }}[i]=0$ holds. This clearly yields the requested tunneled BWT with minimum length. Algorithm 3 gathers the described ideas, and also contains an optimization which allows to build $D_{\text {in }}$ and $D_{\text {out }}$ from copies of the node boundary vector $B$. 


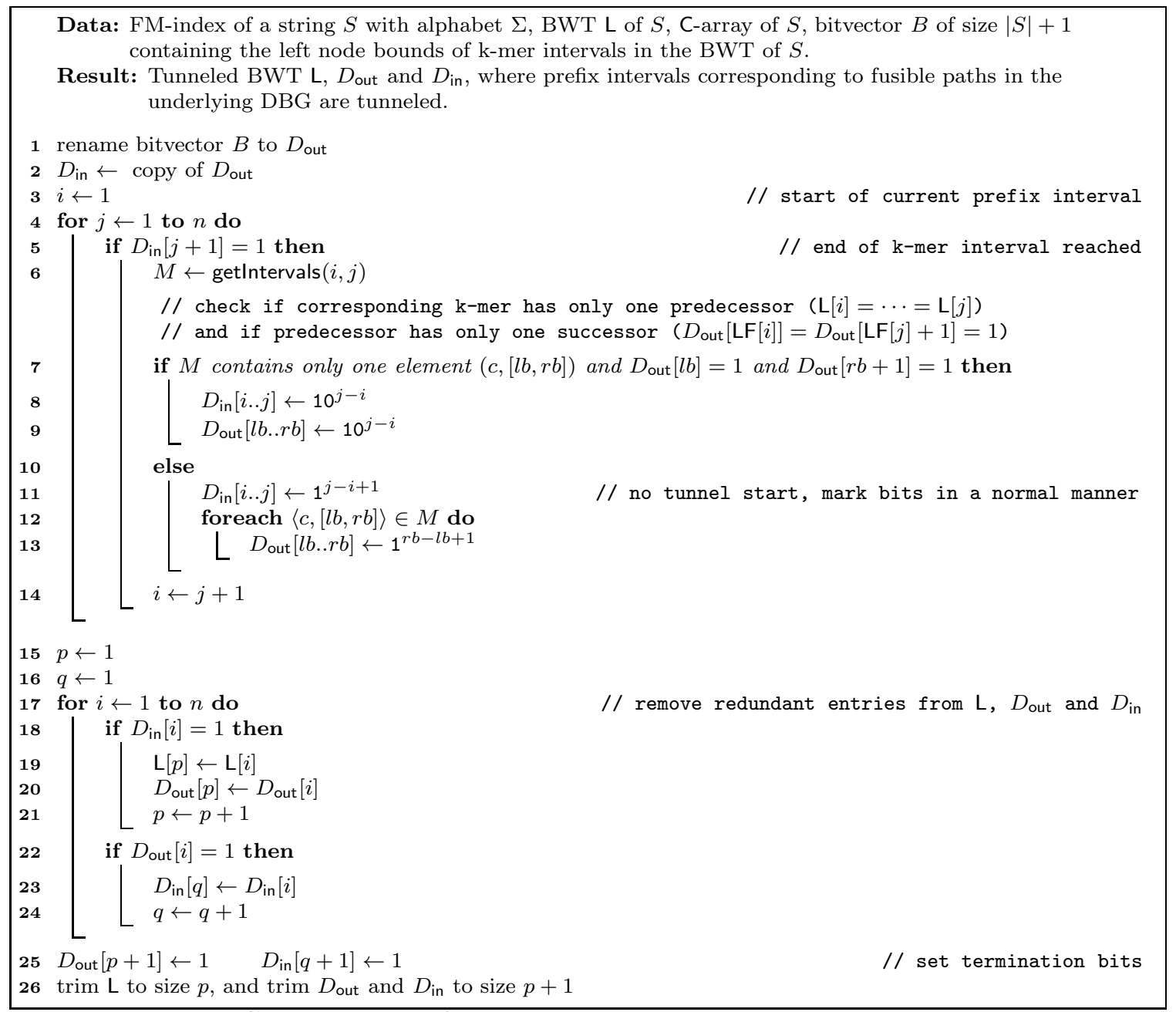

Algorithm 3: Construction of a tunneled BWT using the node boundary bitvector $B$ described in the Section "Retaining the node boundaries of solution". The algorithm requires $O\left(n+m^{*} \log \sigma\right)$ time using the FM-index in form of a wavelet tree, where $n$ is the length of the normal BWT, $m^{*}$ is the number of edges in the edge-reduced de Bruijn graph and $\sigma$ is the alphabet size.

\section{Experimental results}

We implemented the described algorithms using C++ and the sdsl-lite library [22]. All experiments were conducted on a 64 bit Ubuntu 18.04.3 LTS system equipped with two 16-core Intel Xeon E5-2698v3 processors and 256 GB of RAM. All programs were compiled with the $\mathrm{O} 3$ option using $\mathrm{g}++$ (version 7.4.0) and are publicly available 9]. The test data comes from the Pizza \& Chili corpus [10] and the Repetitive corpus [11], where nullbytes were removed from the input files for technical reasons.

Figure 5 shows our main results belonging to de Bruijn graph edge minimization and tunneled FM index construction 3 Not too surprising, the edge reduction ratio for non-repetitive files is moderate ( 5 to $45 \%$ ) but quite high for repetitive files (60 - 95 $\%$ ). Also, the reduction of the data structure size between a normal and a tunneled

\footnotetext{
${ }^{3}$ We refer to an FM-index as a BWT encoded in a wavelet tree.
} 


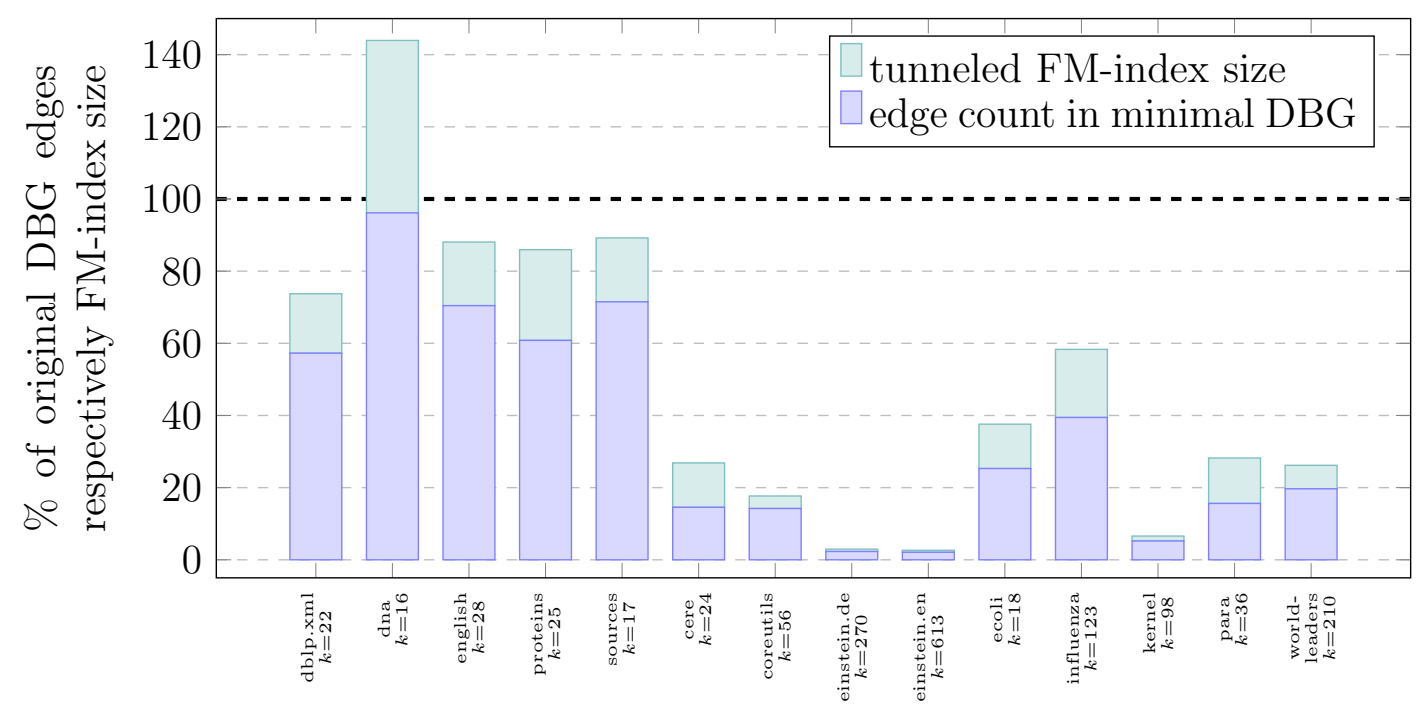

Figure 5: Bar chart for the edge reduction rate of de Bruijn graphs respectively FM-index size reduction using tunneling. The y-tick at $100 \%$ indicates the size of a non-edge-reduced de Bruijn graph resp. a normal FM-index. The order $k$ that minimizes the edge-reduced de Bruijn graph is depicted right beside of each test case name.

FM-index built using the edge minimization algorithm cannot completely keep up with the edge reduction ratio. This is owed to the two additional bitvectors $D_{\text {out }}$ and $D_{\text {in }}$ required to perform a backward step in a tunneled BWT, and also explains why the tunneled FM index for the test file dna is bigger than the conventional FMindex: DNA consists of a 4 character alphabet. As a consequence, encoding DNA in a balanced wavelet tree requires roughly 2 bits per symbol. As a tunneled FM-index requires 2 additional bits per remaining symbol (bitvector $D_{\text {out }}$ and $D_{\text {in }}$ ), and the edge reduction ratio for dna is low, the additional bitvectors size exceeds the benefits of reducing the BWT length.

The edge minimization algorithm typically requires half of the time of a full FMindex construction (suffix array + BWT + wavelet tree), with some variations depending on the number of edges in the edge-minimal DBG of the underlying data. The algorithm, however, is clearly faster than the naive computational approach, which for the sources file required more than 8 hours while the fast algorithm required only 43 seconds-we therefore took a pass on more comparisons with the naive approach. Also, it is surprising that for most files except for some very repetitive inputs the order of the minimal de Bruijn graph is very small, i.e. a number below 30 , but we are not aware of a reason for this.

\section{Conclusion}

We introduced the edge minimization problem and gave an efficient algorithm to it. The usage of an FM-index improves the total run-time of $O\left(n^{2}\right)$ for solving the edge minimization problem to $O(n \log \sigma)$ because of the faster construction of the FM-index compared to the construction of the rotations trie. Furthermore, we showed a deep connection between edge minimization and the "tunneling" compression technique. 
Experiments show that the algorithm is practical and leads to a significant reduction of edges compared to a non-edge-reduced de Bruijn graph. Furthermore, despite the required extra information and excluding one test case, the resulting tunneled FMindex remains smaller than a classical FM-index-especially for very repetitive inputs, the reduction of the data structure size is about $80 \%$.

Therefore, our results are a significant progress on the open problem of finding disjoint prefix intervals in a BWT [8]. They are so in a practical way, but they are also interesting from a theoretical point of view, although we restrict prefix intervals to the special class of $k$-mer prefix intervals. Edge-minimal de Bruijn graphs are interesting in their own right because they constitute graphs with minimum redundancy and may be applicable in other disciplines.

\section{Acknowledgments}

This work was supported by the DFG (OH 53/7-1).

\section{References}

[1] Nicolas G. de Bruijn, "A Combinatorial Problem," Koninklijke Nederlandse Akademie V. Wetenschappe, vol. 49, pp. 758-764, 1946.

[2] Ramana M. Idury and Michael S. Waterman, "A new algorithm for DNA sequence assembly," Journal of Computational Biology, vol. 2, no. 2, pp. 291-306, 1995.

[3] Zamin Iqbal, Mario Caccamo, Isaac Turner, Paul Flicek, and Gil McVean, "De novo assembly and genotyping of variants using colored de bruijn graphs," Nature genetics, vol. 44, pp. 226-32, 2012.

[4] Shoshana Marcus, Hayan Lee, and Michael Schatz, "Splitmem: A graphical algorithm for pan-genome analysis with suffix skips," Bioinformatics, vol. 30, no. 24, pp. 3476-3483, 2014.

[5] Michael Burrows and David J. Wheeler, "A block-sorting lossless data compression algorithm," Tech. Rep. 124, Digital Equipment Corporation, 1994.

[6] Paolo Ferragina and Giovanni Manzini, "Indexing Compressed Text," Journal of the $A C M$, vol. 52, no. 4, pp. 552-581, 2005.

[7] Uwe Baier, "On Undetected Redundancy in the Burrows-Wheeler Transform," in Annual Symposium on Combinatorial Pattern Matching, 2018, CPM '18, pp. 3:1-3:15.

[8] Jarno Alanko, Travis Gagie, Gonzalo Navarro, and Louisa Seelbach Benkner, "Tunneling on Wheeler Graphs," in Proceedings of the 2019 Data Compression Conference, 2019, DCC '19, pp. 122-131.

[9] Uwe Baier, Thomas Büchler, Enno Ohlebusch, and Pascal Weber, "Edge minimization implementation," https://www.uni-ulm.de/fileadmin/website_uni_ulm/iui.inst.190/Forschung/Projekte/seqana

[10] Paolo Ferragina and Gonzalo Navarro, "Pizza \& Chili Corpus," http://pizzachili.dcc.uchile.cl/texts.html, last visited October 2019.

[11] Paolo Ferragina and Gonzalo Navarro, "Repetitive Corpus," http://pizzachili.dcc.uchile.cl/repcorpus.html, last visited October 2019.

[12] Phillip E.C. Compeau, Pavel A. Pevzner, and Glenn Tesler, "How to apply de Bruijn graphs to genome assembly," Nature Biotechnology, vol. 29, no. 11, pp. 987-991, 2011.

[13] Uwe Baier, Timo Beller, and Enno Ohlebusch, "Graphical pan-genome analysis with compressed suffix trees and the Burrows-Wheeler transform," Bioinformatics, vol. 32, no. 4, pp. 497-504, 2016. 
[14] Edward Fredkin, "Trie memory," Communications of the ACM, vol. 3, no. 9, pp. 490-499, 1960.

[15] Peter Weiner, "Linear pattern matching algorithms," in Proc. 14th IEEE Annual Symposium on Switching and Automata Theory, 1973, pp. 1-11.

[16] Timo Beller, Katharina Berger, and Enno Ohlebusch, "Space-Efficient Computation of Maximal and Supermaximal Repeats in Genome Sequences," in Proc. 19th International Symposium on String Processing and Information Retrieval, 2012, SPIRE '12, pp. 99-110.

[17] Enno Ohlebusch, Bioinformatics Algorithms: Sequence Analysis, Genome Rearrangements, and Phylogenetic Reconstruction, Oldenbusch Verlag, 2013.

[18] Paolo Ferragina and Giovanni Manzini, "Opportunistic data structures with applications," in Proc. 41st Annual IEEE Symposium on Foundations of Computer Science, 2000, pp. 390-398.

[19] Roberto Grossi, Ankur Gupta, and Jeffrey S. Vitter, "High-order entropy-compressed text indexes," in Proc. 14th Annual ACM-SIAM Symposium on Discrete Algorithms, 2003, pp. 841-850.

[20] Timo Beller, Simon Gog, Enno Ohlebusch, and Thomas Schnattinger, "Computing the longest common prefix array based on the Burrows-Wheeler transform," Journal of Discrete Algorithms, vol. 18, pp. 22-31, 2013.

[21] Travis Gagie, Gonzalo Navarro, and Simon J. Puglisi, "New algorithms on wavelet trees and applications to information retrieval," Theoretical Computer Science, vol. 426-427, pp. 25-41, 2012.

[22] Simon Gog, "sdsl-lite Library," https://github.com/simongog/sdsl-lite, last visited October 2019. 